\title{
WHITNEY STRATIFICATION OF SETS DEFINABLE IN THE STRUCTURE $\mathbb{R}_{\exp }$
}

\author{
TA LE LOI \\ Department of Mathematics, University of Dalat, Dalat, Vietnam
}

\begin{abstract}
The aim of this paper is to prove that every subset of $\mathbb{R}^{n}$ definable from addition, multiplication and exponentiation admits a stratification satisfying Whitney's conditions a) and $b)$.
\end{abstract}

1. Preliminaries. Let $\mathcal{A}_{n}$ be the smallest ring of real-valued functions on $\mathbb{R}^{n}$ such that:

(a) $\mathcal{A}_{n}$ contains all polynomials, i.e. $\mathbb{R}\left[x_{1}, \ldots, x_{n}\right] \subset \mathcal{A}_{n}$.

(b) $\mathcal{A}_{n}$ is closed under taking exponentiation, i.e. if $f \in \mathcal{A}_{n}$, then $\exp f \in \mathcal{A}_{n}$.

1.1. Definition. Let $\mathcal{D}=\left(\mathcal{D}_{n}\right)_{n \in \mathbb{N}}$ be the smallest class of subsets of Euclidean spaces $\mathbb{R}^{n}, n \in \mathbb{N}$, where $\mathcal{D}_{n}$ is a class of subsets of $\mathbb{R}^{n}$, satisfying the following properties for all $n$ :

(D1) $\mathcal{D}_{n}$ contains all sets of the form $\left\{x \in \mathbb{R}^{n}: f(x)=0\right\}$, where $f \in \mathcal{A}_{n}$.

(D2) If $S, T \in \mathcal{D}_{n}$, then $S \cup T, S \cap T$ and $S \backslash T \in \mathcal{D}_{n}$.

(D3) If $S \in \mathcal{D}_{n+1}$, then $\pi(S) \in \mathcal{D}_{n}$, where $\pi: \mathbb{R}^{n+1} \rightarrow \mathbb{R}^{n}$ is the natural projection.

A set $S$ is called a $\mathcal{D}_{n}$-set iff $S \in \mathcal{D}_{n}$. A $\mathcal{D}$-set is a $\mathcal{D}_{n}$-set for some $n \in \mathbb{N}$. A function $f: S \rightarrow \mathbb{R}$ is called a $\mathcal{D}$-function iff its graph is a $\mathcal{D}$-set.

Rem ark. The class $\mathcal{D}$ contains all semi-algebraic sets. A $\mathcal{D}$-set, in general, is not subanalytic (e.g. $\{(x, y): x>0, y=\exp (-1 / x)\}$ ). If $f$ is a $\mathcal{D}$-function, then so is $\exp f$. If, in addition, $f>0$, then $\log f, f^{\alpha}(\alpha \in \mathbb{R})$ are $\mathcal{D}$-functions. The closure, the interior and the boundary in $\mathbb{R}^{n}$ of a $\mathcal{D}_{n}$-set are $\mathcal{D}_{n}$-sets.

The following theorem is due to Wilkie [9], [10], which is an essential result for the class $\mathcal{D}$.

1991 Mathematics Subject Classification: 14P99, 32B25.

Key words and phrases: $\mathcal{D}$-sets, Whitney stratification.

The paper is in final form and no version of it will be published elsewhere. 
1.2. TheOREM (Wilkie). Let $S \subset \mathbb{R}^{n}$ be a $\mathcal{D}$-set. Then there exists $f \in \mathcal{A}_{n+m}$, for some $m \in \mathbb{N}$, such that $S=\pi\left(f^{-1}(0)\right)$, where $\pi: \mathbb{R}^{n} \times \mathbb{R}^{m} \rightarrow \mathbb{R}^{n}$ is the natural projection.

Combining the theorem with a Khovanskiu result on fewnomials [4] it follows that every $\mathcal{D}$-set has only finitely many connected components.

1.3. Definition (cf. [2]). (i) A map $f: S \rightarrow \mathbb{R}^{m}$ with $S \subset \mathbb{R}^{n}$ is called a $\mathcal{D}$-map if its graph belongs to $\mathcal{D}_{n+m}$. In this case it is called $\mathcal{D}$-analytic if there is an open neighborhood $U$ of $S$ in $\mathbb{R}^{n}, U \in \mathcal{D}_{n}$ and an analytic $\mathcal{D}$-map $F: U \rightarrow \mathbb{R}^{m}$ such that $\left.F\right|_{S}=f$.

(ii) $\mathcal{D}_{n}$-analytic cells in $\mathbb{R}^{n}$ are defined by induction on $n: \mathcal{D}_{1}$-analytic cells are points $\{r\}$ or open intervals $(a, b),-\infty \leq a<b \leq+\infty$. If $C$ is a $\mathcal{D}_{n}$-analytic cell and $f, g: C \rightarrow \mathbb{R}$ are $\mathcal{D}$-analytic such that $f<g$, then

$$
\begin{aligned}
(f, g) & :=\{(x, r) \in C \times \mathbb{R}: f(x)<r<g(x)\}, \\
(-\infty, f) & :=\{(x, r) \in C \times \mathbb{R}: r<f(x)\}, \\
(g,+\infty) & :=\{(x, r) \in C \times \mathbb{R}: g(x)<r\}, \\
\Gamma(f) & :=\text { graph } f \text { and } C \times \mathbb{R} \text { are } \mathcal{D}_{n+1} \text {-analytic cells. }
\end{aligned}
$$

(iii) A $\mathcal{D}$-analytic decomposition of $\mathbb{R}^{n}$ is defined by induction on $n$ : A $\mathcal{D}$-analytic decomposition of $\mathbb{R}^{1}$ is a finite collection of intervals and points $\left\{\left(-\infty, a_{1}\right), \ldots,\left(a_{k},+\infty\right)\right.$, $\left.\left\{a_{1}\right\}, \ldots,\left\{a_{k}\right\}\right\}$, where $a_{1}<\ldots<a_{k}, k \in \mathbb{N}$. A $\mathcal{D}$-analytic decomposition of $\mathbb{R}^{n+1}$ is a finite partition of $\mathbb{R}^{n+1}$ into $\mathcal{D}_{n+1}$-analytic cells $C$ such that the collection of all the projections $\pi(C)$ is a $\mathcal{D}$-analytic decomposition of $\mathbb{R}^{n}$ (here $\pi: \mathbb{R}^{n+1} \rightarrow \mathbb{R}^{n}$ is the natural projection).

We say that a decomposition partitions $S$ if $S$ is a union of some cells of the decomposition.

1.4. Theorem (van den Dries \& Miller). $\left(\mathrm{I}_{n}\right)$ For $S_{1}, \ldots, S_{k} \in \mathcal{D}_{n}$ there is a $\mathcal{D}$ analytic decomposition of $\mathbb{R}^{n}$ partitioning $S_{1}, \ldots, S_{k}$.

$\left(\mathrm{II}_{n}\right)$ For every fuction $f: S \rightarrow \mathbb{R}, S \in \mathcal{D}_{n}$, there is a $\mathcal{D}$-analytic decomposition of $\mathbb{R}^{n}$ partitioning $S$ such that for each cell $C \subset S$ of the decomposition, the restriction $\left.f\right|_{C}$ is $\mathcal{D}$-analytic.

For the proof see [1], [2] or [6].

1.5. Corollary. Let $S_{1}, \ldots, S_{k}$ be $\mathcal{D}_{n}$-sets. Then there is an analytic stratification of $\mathbb{R}^{n}$ compatible with $S_{1}, \ldots, S_{k}$. Precisely, there is a finite family $\left\{\Gamma_{\alpha}^{d}\right\}$ of subsets of $\mathbb{R}^{n}$ such that:

(S1) $\Gamma_{\alpha}^{d}$ are disjiont, $\mathbb{R}^{n}=\bigcup_{\alpha, d} \Gamma_{\alpha}^{d}$ and $S_{i}=\bigcup\left\{\Gamma_{\alpha}^{d}: \Gamma_{\alpha}^{d} \cap S_{i} \neq \emptyset\right\}, i=1, \ldots, k$.

(S2) Each $\Gamma_{\alpha}^{d}$ is a $\mathcal{D}_{n}$-analytic cell of dimension $d$.

(S3) $\overline{\Gamma_{\alpha}^{d}} \backslash \Gamma_{\alpha}^{d}$ is a union of some cells $\Gamma_{\beta}^{e}$ with $e<d$.

1.6. Corollary. Let $f: \mathbb{R} \rightarrow \mathbb{R}$ be a $\mathcal{D}$-function. Then the limits $\lim _{+\infty} f, \lim _{-\infty} f$, $\lim _{c^{+}} f$ and $\lim _{c^{-}} f(c \in \mathbb{R})$ exist in $\mathbb{R} \cup\{-\infty,+\infty\}$.

1.7. Corollary (curve selecting lemma). Let $S \subset \mathbb{R}^{n}$ be a $\mathcal{D}$-set and $a \in \bar{S}$ be a nonisolated point of $S$. Then there exists an analytic $\mathcal{D}$-map $\gamma:(0,1) \rightarrow S$ such that $\lim _{0^{+}} \gamma=a$. 
For the proof of the corollaries see [5], [6].

Remark. The class $\mathcal{D}$ shares many nice properties with those of semi-algebraic sets (see [1], [5], [7]).

2. Whitney stratification. In this section we prove the existence of the Whitney stratification of $\mathcal{D}$-sets. The proof is inspired by that of Łojasiewicz [8] for semianalytic sets.

Let $G_{k}\left(\mathbb{R}^{n}\right)$ denote the Grassmannian of $k$-dimensional vector subspaces of $\mathbb{R}^{n}$. Let $\langle$,$\rangle denote the scalar product with respect to the canonical base of \mathbb{R}^{n}$. Then $G_{k}\left(\mathbb{R}^{n}\right)$ can be identified with the set of all $n \times n$ matrices $A \in \operatorname{Mat}(n, n)$ with $A^{2}=A,{ }^{t} A=A$ and trace $A=k$. Therefore $G_{k}\left(\mathbb{R}^{n}\right)$ is an algebraic subset of $\mathbb{R}^{n^{2}}$. So it is a $\mathcal{D}$-set.

2.1. Proposition. Let $X$ be an analytic submanifold of $\mathbb{R}^{n}$ which is also a $\mathcal{D}$-set. Suppose that $\phi_{1}, \ldots, \phi_{k}: X \rightarrow \mathbb{R}^{n}$ are analytic $\mathcal{D}$-maps such that for all $x$ in $X$ the vectors $\phi_{1}(x), \ldots, \phi_{k}(x)$ generate a $k$-dimensional vector subspace $\Phi(x)$ of $\mathbb{R}^{n}$. Then the map $\Phi: X \rightarrow G_{k}\left(\mathbb{R}^{n}\right)$ is a $\mathcal{D}$-map. Consequently, if $X$ is of dimension $k$, then the map

$$
\mathcal{T}_{X}: X \rightarrow G_{k}\left(\mathbb{R}^{n}\right) \quad \text { defined by } \quad \mathcal{T}_{X}(x)=T_{X, x}
$$

(where $T_{X, x}$ denotes the tangent space of $X$ at $x$ ) is a $\mathcal{D}$-map.

Pr o of. Since $\Phi(x)$ is identified with the orthogonal projection of $\mathbb{R}^{n}$ onto $\Phi(x)$,

$$
\Phi(x) \cdot h=\sum_{i=1}^{k} a_{i}(x, h) \phi_{i}(x)
$$

where

$$
\left(\begin{array}{c}
a_{1}(x, h) \\
\vdots \\
a_{k}(x, h)
\end{array}\right)=A^{-1}(x)\left(\begin{array}{c}
\left\langle h, \phi_{1}(x)\right\rangle \\
\vdots \\
\left\langle h, \phi_{k}(x)\right\rangle
\end{array}\right)
$$

with $A(x)$ being the $k \times k$ matrix $\left(\left\langle\phi_{i}(x), \phi_{j}(x)\right\rangle\right)$. So the coefficients of $\Phi$ are $\mathcal{D}$-functions. This implies that $\Phi$ is a $\mathcal{D}$-map.

Let $C \subset \mathbb{R}^{n}$ be a $\mathcal{D}$-analytic cell of dimension $k$. Then, by Definition $1.3, C$ can be parametrized by an analytic $\mathcal{D}$-map $\phi: U \rightarrow \mathbb{R}^{n}$, where $U$ is an open $\mathcal{D}$-set of $\mathbb{R}^{k}$. Put $\phi_{i}(x)=\left(\partial \phi / \partial y_{i}\right)\left(\phi^{-1}(x)\right), x \in C, i=1, \ldots, k$. By the first part of the proposition, $\mathcal{T}_{C}$ is a $\mathcal{D}$-map. If $X$ is of dimension $k$, then, by Theorem $1.4, X$ can be partitioned into finitely many cells $C_{j}$. It is easy to see that

$$
\operatorname{graph} \mathcal{T}_{X}=\left\{(x, T): x \in X, T=T_{X, x}\right\}=X \times G_{k}\left(\mathbb{R}^{n}\right) \cap\left(\bigcup_{j: \operatorname{dim} C_{j}=k} \overline{\operatorname{graph} \mathcal{T}_{C_{j}}}\right)
$$

Thus $\mathcal{T}_{X}$ is a $\mathcal{D}$-map.

2.2. Definition. Let $X, Y$ be analytic submanifolds of $\mathbb{R}^{n}$ of dimensions $k$ and $l$ respectively. Suppose that $X \cap Y=\emptyset$ and $Y \subset \bar{X}$. Let $y \in Y$. We say that $(X, Y)$ satisfies Whitney's condition a) at $y$ if the following condition is satisfied:

a) For any sequence $\left(x_{\nu}\right)_{\nu \in \mathbb{N}}$ of points of $X$ with $\lim x_{\nu}=y$, if $\lim T_{X, x_{\nu}}=\tau$ in $G_{k}\left(\mathbb{R}^{n}\right)$, then $\tau \supset T_{Y, y}$. 
We say that $(X, Y)$ satisfies Whitney's condition b) at $y$ iff

b) For any pair of sequences $\left(x_{\nu}\right)_{\nu \in \mathbb{N}}, x_{\nu} \in X$, and $\left(y_{\nu}\right)_{\nu \in \mathbb{N}}, y_{\nu} \in Y$, with $\lim x_{\nu}=$ $\lim y_{\nu}=y$, if $\lim T_{X, x_{\nu}}=\tau$ and the sequence of lines $\mathbb{R}\left(x_{\nu}-y_{\nu}\right)$ has a limit $\lambda$ in $G_{1}\left(\mathbb{R}^{n}\right)$, then $\tau \supset \lambda$.

2.3. Re mark. Let $\delta: G_{l}\left(\mathbb{R}^{n}\right) \times G_{k}\left(\mathbb{R}^{n}\right) \rightarrow \mathbb{R}$ be the function defined by

$$
\delta(E, F)=\sup \{d(x, F): x \in E,\|x\|=1\}, \quad E \in G_{l}\left(\mathbb{R}^{n}\right), F \in G_{k}\left(\mathbb{R}^{n}\right) \quad(l \leq k) .
$$

Then $\delta$ is semialgebraic (so it is a $\mathcal{D}$-function) and $\delta(E, F)=0 \Leftrightarrow E \subset F$. If $K \subset F$ is a vector subspace, then $\delta(E, F) \leq \delta(E, K)$. If $E=\operatorname{graph} \eta$ and $F=\operatorname{graph} \theta$, where $\eta, \theta: \mathbb{R}^{p} \rightarrow \mathbb{R}^{q}$ are linear maps $(p+q=n)$, then $\delta(E, F) \leq\|\theta-\eta\|$.

2.4. Proposition. Under the notation of Def. 2.2, let $W_{\mathrm{a}}(X, Y)\left(\operatorname{resp} . W_{\mathrm{b}}(X, Y)\right)$ be the set of points of $Y$ at which $(X, Y)$ satisfies Whitney's condition a) (resp. b)). Then $W_{\mathrm{a}}(X, Y)$ and $W_{\mathrm{b}}(X, Y)$ are $\mathcal{D}$-sets.

Proof. We have

$$
\begin{aligned}
W_{\mathrm{a}}(X, Y) & =\left\{y \in Y: \forall \tau \in G_{k}\left(\mathbb{R}^{n}\right),(y, \tau) \in \overline{\operatorname{graph} \mathcal{T}_{X}} \Rightarrow \tau \supset T_{Y, y}\right\} \\
& =\left\{y \in Y: \forall \tau \in G_{k}\left(\mathbb{R}^{n}\right),(y, \tau) \in \overline{\operatorname{graph} \mathcal{T}_{X}} \Rightarrow \delta\left(T_{Y, y}, \tau\right)=0\right\} .
\end{aligned}
$$

By Proposition 2.1, Remark 2.3 and Definition 1.1, $W_{\mathrm{a}}(X, Y)$ is a $\mathcal{D}$-set.

Similarly, let $V=\left\{(x, T, y, d) \in \mathcal{T}_{X} \times Y \times G_{1}\left(\mathbb{R}^{n}\right): d=\mathbb{R}(x-y)\right\}$. By Proposition 2.1 the map $X \times Y \ni(x, y) \mapsto \mathbb{R}(x-y) \in G_{1}\left(\mathbb{R}^{n}\right)$ is a $\mathcal{D}$-map. So $V$ is a $\mathcal{D}$-set. Then

$$
W_{\mathrm{b}}(X, Y)=\left\{y \in Y: \forall \tau \in G_{k}\left(\mathbb{R}^{n}\right), \forall \lambda \in G_{1}\left(\mathbb{R}^{n}\right),(y, \tau, y, \lambda) \in \bar{V} \Rightarrow \delta(\lambda, \tau)=0\right\}
$$

is also a $\mathcal{D}$-set.

2.5. TheOrem. Let $X, Y$ be analytic submanifolds of $\mathbb{R}^{n}$ which are $\mathcal{D}$-sets. Suppose that $X \cap Y=\emptyset$ and $Y \subset \bar{X}$. Then

$$
\operatorname{dim}\left(Y \backslash W_{\mathrm{a}}(X, Y)\right)<\operatorname{dim} Y \quad \text { and } \quad \operatorname{dim}\left(Y \backslash W_{\mathrm{b}}(X, Y)\right)<\operatorname{dim} Y .
$$

To prove this theorem we prepare some lemmas.

2.6. Lemma (definable selection). Let $S \subset \mathbb{R}^{p} \times \mathbb{R}^{m}$ be a $\mathcal{D}$-set and let $\pi: \mathbb{R}^{p} \times \mathbb{R}^{m} \rightarrow$ $\mathbb{R}^{p}$ be the natural projection. Then there exists a $\mathcal{D}$-map $\varrho: \pi(S) \rightarrow \mathbb{R}^{p} \times \mathbb{R}^{m}$ such that $\pi(\varrho(x))=x$ for all $x \in \pi(S)$.

Pr o of (cf. [1, Ch. 8, Prop. (1.2)]). Clearly, it is sufficient to prove the lemma for $m=1$. Moreover, by Theorem 1.4, we may assume that $S$ is a cell. We define $\varrho$ as follows:

If $S=(f, g)$, where $f, g: \pi(S) \rightarrow \mathbb{R}$ are $\mathcal{D}$-functions, let $\varrho(x)=\left(x, \frac{1}{2}(f(x)+g(x))\right)$.

If $S=(-\infty, g)$, where $g: \pi(S) \rightarrow \mathbb{R}$ is a $\mathcal{D}$-function, let $\varrho(x)=(x, g(x)-1)$.

If $S=(f,+\infty)$, where $f: \pi(S) \rightarrow \mathbb{R}$ is a $\mathcal{D}$-function, let $\varrho(x)=(x, f(x)+1)$.

If $S=\Gamma(f)$, where $f: \pi(S) \rightarrow \mathbb{R}$ is a $\mathcal{D}$-function, let $\varrho(x)=(x, f(x))$.

If $S=\pi(S) \times \mathbb{R}$, let $\varrho(x)=(x, 0)$.

2.7. Lemma (half wing). Let $S, V \subset \mathbb{R}^{p} \times \mathbb{R}^{q}$ be $\mathcal{D}$-sets, $S \cap V=\emptyset$ and $V \subset \bar{S}$. Suppose that $V$ is open in $\mathbb{R}^{p}$, where $\mathbb{R}^{p} \equiv \mathbb{R}^{p} \times O \subset \mathbb{R}^{p} \times \mathbb{R}^{q}$. Let $\pi: \mathbb{R}^{p} \times \mathbb{R}^{q} \rightarrow \mathbb{R}^{p}$ be the natural projection. Then there are an open $\mathcal{D}$-set $U$ in $V, r>0$ and an analytic $\mathcal{D}$-map $\bar{\theta}:(0, r) \times U \rightarrow S$ such that $\bar{\theta}(t, y)=(y, \theta(t, y))$ and $\|\theta(t, y)\| \leq t$ for all $(t, y) \in(0, r) \times U$. 
Proof. Let

$$
A=\{(t, y, x): 0<t<1, y \in V, x \in S,\|x-y\| \leq t, \pi(x)=y\} .
$$

Then $A$ is a $\mathcal{D}$-set. If $\pi_{1}$ is the projection defined by $\pi_{1}(t, y, x)=y$, then $\pi_{1}(A)$ is dense in $V$ (so is of dimension $p$ ). Indeed, let $y_{0} \in V$ and $\delta>0$. Then there is $\delta^{\prime}, 0<\delta^{\prime}<\min \left(\frac{1}{2}, \delta\right)$ such that $B\left(y_{0}, \delta^{\prime}\right) \cap \mathbb{R}^{p} \times O \subset V$. Since $y_{0} \in \bar{S} \backslash S$, there is $x \in B\left(y_{0}, \delta^{\prime}\right) \cap S$. Then $y=\pi(x) \in B\left(y_{0}, \delta^{\prime}\right) \cap \pi_{1}(A)$.

Now, let $\pi_{2}$ be the projection defined by $\pi_{2}(t, y, x)=(t, y)$. Put

$$
\pi_{2}(A)_{y}=\left\{t \in(0,1):(t, y) \in \pi_{2}(A)\right\}, \quad \varepsilon(y)=\inf \pi_{2}(A)_{y}, \quad y \in \pi_{1}(A) .
$$

Then $\varepsilon$ is a $\mathcal{D}$-function and $\varepsilon(y)>0 \Rightarrow(0, \varepsilon(y)) \cap \pi_{2}(A)_{y}=\emptyset$.

Claim 1. $\operatorname{dim}\left\{y \in \pi_{1}(A): \varepsilon(y)>0\right\}<p$.

Conversely, suppose that the dimension equals $p$. Then, by Theorem 1.4, there is an open $\mathcal{D}$-set $W$ in $\mathbb{R}^{p}, W \subset \pi_{1}(A)$ on which $\varepsilon$ is analytic and $\varepsilon>c$ for some $c>0$. Let $y_{0} \in W$ and $\delta \in \mathbb{R}, 0<\delta<c$, such that $B\left(y_{0}, \delta\right) \cap \mathbb{R}^{p} \times O \subset W$. Then $\|\pi(x)-y\|>c>\delta$, $\forall y \in B\left(y_{0}, \delta\right) \cap \mathbb{R}^{p} \times O, \forall x \in S$ with $\pi(x)=y$. This contradicts the argument above. The claim is verified.

Claim 2. If $y \in \pi_{1}(A), \varepsilon(y)=0$, then $\pi_{2}(A)_{y} \supset(0,1)$.

Since $\pi_{2}(A)_{y}$ is a nonempty $\mathcal{D}$-set and $0 \in \overline{\pi_{2}(A)} \backslash \backslash \pi_{2}(A)_{y}$, there is $\delta>0$ such that $(0, \delta) \subset \pi_{2}(A)_{y}$, i.e. there is $x$ in $S, \pi(x)=y$ and $\|x-y\| \leq \delta$. So for every $t \in(0,1), t \geq \delta$, $\|x-y\| \leq t$, i.e. $t \in \pi_{2}(A)_{y}$. The claim follows.

Let $V_{1}=\left\{y \in \pi_{1}(A): \varepsilon(y)=0\right\}$. Then, from Claim 1, $\operatorname{dim} V_{1}=p$ and, from Claim 2, $\pi_{2}(A) \supset(0,1) \times V_{1}$. By the definable selection lemma there is a $\mathcal{D}$-map $\varrho:(0,1) \times V_{1} \rightarrow A$ such that $\varrho(t, y)=(t, y, \bar{\theta}(t, y))$. That means $\bar{\theta}:(0,1) \times V_{1} \rightarrow S$ satisfies $\pi(\bar{\theta}(t, y))=y$ and $\|\bar{\theta}(t, y)-y\| \leq t$, i.e. $\bar{\theta}(t, y)=(y, \theta(t, y))$ and $\|\theta(t, y)\| \leq t$.

By Theorem 1.4, with $t$ regarded as the last coordinate, $(0,1) \times V_{1}$ can be partitioned into cells such that the restriction of $\theta$ to each of the cells is analytic. Let $C$ be a cell of the partition with $\operatorname{dim} \pi_{3}(C)=p$ (here $\pi_{3}(t, y)=y$ ). By the definition of cells, there is an analytic $\mathcal{D}$-function $f: \pi_{3}(C) \rightarrow \mathbb{R}, f>0$, such that $(0, f)$ is a cell of the partition. This implies that there are an open $\mathcal{D}$-set $U$ in $\pi_{3}(C)$ and $r>0$ such that $f>r$ on $U$. Therefore, $\theta$ is analytic on $(0, r) \times U$. This finishes the proof of the lemma.

2.8. Lemma. Under the notation of the above lemma, for every $c>0$ there is $\left(t_{c}, y_{c}\right) \in$ $(0, r) \times U$ such that $\left\|d_{y} \theta\left(t_{c}, y_{c}\right)\right\|<c$.

Proof. Let $\theta=\left(\theta_{1}, \ldots, \theta_{q}\right)$. For each $i \in\{1, \ldots, q\}$, let

$$
A_{i}=\left\{(t, y) \in(0, r) \times U:\left\|d_{y} \theta_{i}(t, y)\right\|<c / \sqrt{q}\right\} .
$$

Then $A_{1}, \ldots, A_{q}$ are open $\mathcal{D}$-sets.

Claim: $0 \times U \subset \bar{A}_{i} \backslash A_{i}$, for all $i \in\{1, \ldots, q\}$.

Let $y_{0} \in U, \delta \in \mathbb{R}, 0<\delta<\min \left(d\left(y_{0},{ }^{c} U\right), c /(2 \sqrt{q})\right)$, and $t \in(0, r), 0<t<\delta^{2} / 2$. For each $i$ consider the function

$$
\psi_{i}: U \ni y \mapsto \theta_{i}(t, y)+\left\|y-y_{0}\right\|^{2}-t \in \mathbb{R} .
$$


By Lemma 2.6, $-t \leq \theta_{i}(t, y) \leq t$ for all $y \in U$. We have

$$
\begin{aligned}
\psi_{i}(y) & \geq-2 t+\delta^{2}>0 \quad \forall y \in U,\left\|y-y_{0}\right\|=\delta, \\
\psi_{i}\left(y_{0}\right) & =\theta_{i}\left(t, y_{0}\right)-t \leq 0 .
\end{aligned}
$$

Therefore $\psi_{i}$ has a critical point in $B\left(y_{0}, \delta\right)$, i.e. there is a $\bar{y} \in B\left(y_{0}, \delta\right)$ such that

$$
d_{y} \theta_{i}(t, \bar{y})+2\left(\bar{y}-y_{0}\right)=0 \text {. }
$$

This implies $\left\|d_{y} \theta_{i}(t, \bar{y})\right\|<2 \delta<c / \sqrt{q}$. The claim is verified.

Now, let $\left(A_{i}\right)_{y}=\left\{t \in(0, r):(t, y) \in A_{i}\right\}$. For each $i \in\{1, \ldots, q\}$ define

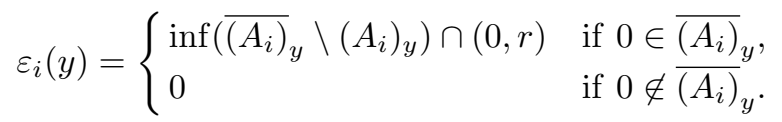

Then $\varepsilon_{i}$ is a $\mathcal{D}$-function on $U$ and $\varepsilon_{i}(y) \neq 0 \Leftrightarrow\left(A_{i}\right)_{y} \supset\left(0, \varepsilon_{i}(y)\right)$.

Since $0 \times U \subset \bar{A}_{i} \backslash A_{i}, \operatorname{dim}\left\{y \in U: \varepsilon_{i}(y)=0\right\}<p$. (If not, then there is an open $\mathcal{D}$-set $U_{i}$ in $U$ on which $\varepsilon_{i} \equiv 0$. Let $\alpha_{i}: U_{i} \rightarrow \mathbb{R}$ be defined by $\alpha_{i}(y)=\inf \left(A_{i}\right)_{y}$. Then $\alpha_{i}$ is a $\mathcal{D}$-function, $\alpha_{i}>0$ and $\left(0, \alpha_{i}(y)\right) \cap\left(A_{i}\right)_{y}=\emptyset$. By Theorem 1.4 there are an open cell $V_{i} \subset U_{i}$ and $M>0$ such that $\left.\alpha_{i}\right|_{V_{i}} \geq M$. This implies $O \times V_{i} \not \subset \bar{A}_{i} \backslash A_{i}$, a contradiction.) So $U \backslash \bigcup_{i=1}^{q}\left\{y \in U: \varepsilon_{i}(y)=0\right\}$ is of dimension $p$. For each $y$ in this set, $\varepsilon(y):=\min _{1 \leq i \leq q} \varepsilon_{i}(y)>0$. Thus $(\varepsilon(y) / 2, y) \in A_{i}, \forall i \in\{1, \ldots, q\}$, i.e. this point satisfies the demand of the lemma.

2.9. Lemma. Let $X, Y$ be analytic submanifolds of $\mathbb{R}^{n}$ of dimensions $k$ and $p$ respectively. Suppose that $X, Y$ are $\mathcal{D}$-sets, $X \cap Y=\emptyset, Y \subset \bar{X}$ and $Y$ is open in $\mathbb{R}^{p} \equiv \mathbb{R}^{p} \times O$. Let $\pi: \mathbb{R}^{n} \equiv \mathbb{R}^{p} \times \mathbb{R}^{q} \rightarrow \mathbb{R}^{p}$ be the natural projection. Let $W_{\mathrm{b}^{\prime}}(X, Y)$ be the set of points $y$ of $Y$ where $(X, Y)$ satisfies the following condition:

$\left.\mathrm{b}^{\prime}\right)$ For any sequence $\left(x_{\nu}\right)_{\nu \in \mathbb{N}}$ of points of $X$ with $\lim x_{\nu}=y$, if $\lim T_{X, x_{\nu}}=\tau \in$ $G_{k}\left(\mathbb{R}^{n}\right)$ and $\lim \mathbb{R}\left(x_{\nu}-\pi\left(x_{\nu}\right)\right)=\lambda \in G_{1}\left(\mathbb{R}^{n}\right)$, then $\tau \supset \lambda$.

Then $W_{\mathrm{b}^{\prime}}(X, Y)$ is a $\mathcal{D}$-set and $W_{\mathrm{a}}(X, Y) \cap W_{\mathrm{b}^{\prime}}(X, Y) \subset W_{\mathrm{b}}(X, Y)$.

Proof. Similarly to the proof of Proposition 2.4 it is easy to prove that $W_{\mathrm{b}^{\prime}}(X, Y)$ is a $\mathcal{D}$-set. We prove the second part of the lemma.

Let $y \in W_{\mathrm{a}}(X, Y) \cap W_{\mathrm{b}^{\prime}}(X, Y)$. Define

$$
F\left\{(x, T, y, d): x \in X, T=T_{X, x}, y \in Y, d=\mathbb{R}(x-y)\right\} .
$$

Let $(y, \tau, y, \lambda) \in \bar{F}$. It suffices to prove that $\lambda \subset \tau$.

By Corollary 1.7 there is a continuous $\mathcal{D}$-map

$$
\gamma:[0,1] \rightarrow \mathbb{R}^{n} \times G_{k}\left(\mathbb{R}^{n}\right) \times \mathbb{R}^{n} \times G_{1}\left(\mathbb{R}^{n}\right)
$$

such that $\gamma$ is analytic on $(0,1), \gamma(0)=(y, \tau, y, \lambda)$ and for all $t$ in $(0,1], \gamma(t)=\left(\gamma_{1}(t)\right.$, $\left.T_{X, \gamma_{1}(t)}, \gamma_{2}(t), \mathbb{R}\left(\gamma_{1}(t)-\gamma_{2}(t)\right)\right)$ with $\gamma_{1}(t) \in X, \gamma_{2}(t) \in Y$.

Since $(X, Y)$ satisfies condition a) at $y, \tau \supset T_{Y, y}$.

Since $(X, Y)$ satisfies condition $\left.\mathrm{b}^{\prime}\right)$ at $y, \tau \supset \lim _{0+} \mathbb{R}\left(\gamma_{1}(t)-\pi\left(\gamma_{1}(t)\right)\right)$ (this limit exists by Corollary 1.6). This implies $\lambda=\lim _{0^{+}} \mathbb{R}\left(\gamma_{1}(t)-\gamma_{2}(t)\right) \subset \tau$ because $\mathbb{R}\left(\gamma_{1}(t)-\gamma_{2}(t)\right)$ is contained in the vector subspace spanned by $\mathbb{R}\left(\gamma_{1}(t)-\pi\left(\gamma_{1}(t)\right)\right)$ and $\mathbb{R}\left(\gamma_{2}(t)-\pi\left(\gamma_{1}(t)\right)\right) \subset$ $T_{Y, y}=\mathbb{R}^{p} \times O$. 
2.10. LemmA. Let $X$ be a $\mathcal{D}$-set which is also an analytic submanifold of $\mathbb{R}^{n}$. Let $y \in \bar{X} \backslash X$ and $\gamma:(0, \varepsilon) \rightarrow X(\varepsilon>0)$ be an analytic $\mathcal{D}$-function with $\lim _{0^{+}} \gamma=y$. Then $\lim _{t \rightarrow 0^{+}} \delta\left(\mathbb{R}(\gamma(t)-y), T_{X, \gamma(t)}\right)=0$.

Pr o of. Since $y \notin X, \gamma(t) \not \equiv$ const. So $\gamma^{\prime} \not \equiv 0$. Moreover, $\gamma^{\prime}$ is a $\mathcal{D}$-map, and reducing $\varepsilon$, we can assume that $\gamma^{\prime} \neq 0$. By Corollary 1.6 the $\operatorname{limit} \lim _{0^{+}} \gamma^{\prime} /\left\|\gamma^{\prime}\right\|$ exists. This implies the curve $C=\{y\} \cup \gamma(0, \varepsilon)$ is a $C^{1}$ curve. So $\lim _{0^{+}} \delta\left(\mathbb{R}(\gamma(t)-y), \mathbb{R} \gamma^{\prime}(t)\right)=0$. But $\gamma^{\prime}(t) \in T_{X, \gamma(t)}, \forall t \in(0, \varepsilon)$. Thus $\lim _{0^{+}} \delta\left(\mathbb{R}(\gamma(t)-y), T_{X, \gamma(t)}\right)=0$.

Proof of Theorem 2.5. Let $p=\operatorname{dim} Y$. By Theorem 1.4, $Y$ can be partitioned into finitely many cells $C_{j}$. It is therefore sufficient to prove that for any $j$ with $\operatorname{dim} C_{j}=p$, both $\operatorname{dim}\left(C_{j} \backslash W_{\mathrm{a}}(X, Y)\right)$ and $\operatorname{dim}\left(C_{j} \backslash W_{\mathrm{b}}(X, Y)\right)$ are smaller than $p$.

Moreover, the Whitney conditions are of a local nature and invariant under analytic isomorphisms, and from the definition of cells, we may assume that $Y$ is an open $\mathcal{D}$-set in $\mathbb{R}^{p} \equiv \mathbb{R}^{p} \times O \subset \mathbb{R}^{p} \times \mathbb{R}^{q}(p+q=n)$.

$$
\begin{aligned}
& \text { Proof of } \operatorname{dim}\left(Y \backslash W_{\mathrm{a}}(X, Y)\right)<p \text {. Define } \\
& \qquad \phi(y, t)=\sup \left\{\delta\left(\mathbb{R}^{p}, T_{X, x}\right): x \in X,\|x-y\| \leq t\right\}, \quad y \in Y, t>0 .
\end{aligned}
$$

Then $\phi$ is a $\mathcal{D}$-function. For each $y \in Y, \phi(y, \cdot)$ is a bounded $\mathcal{D}$-function with respect to t. Then, by Corollary 1.6, there exists $\lim _{t \rightarrow 0^{+}} \phi(y, t)=f(y) \in \mathbb{R}, \forall y \in Y$. Note that $f: Y \rightarrow \mathbb{R}$ is a $\mathcal{D}$-function and $f(y) \neq 0 \Leftrightarrow y \in W_{\mathrm{a}}(X, Y)$.

Suppose, contrary to our assertion, that $\operatorname{dim}\left(Y \backslash W_{\mathrm{a}}(X, Y)\right)=p$. Then, from Theorem 1.4, there are an open $\mathcal{D}$-set $V$ in $Y$ and $c>0$ such that $f>c$ on $V$. Let $S=\left\{x \in X: \delta\left(\mathbb{R}^{p}, T_{X, x}\right) \geq c\right\}$. Then $V \subset \bar{S} \backslash S$. By Lemma 2.7, there are an open $\mathcal{D}$-set $U \subset V, r>0$ and an analytic $\mathcal{D}$-map $\bar{\theta}:(0, r) \times U \rightarrow S$ such that $\bar{\theta}(t, y)=(y, \theta(t, y))$ and $\|\theta(t, y)\| \leq t$, for all $(t, y) \in(0, r) \times U$.

From Lemma 2.8, there exists $\left(t_{c}, y_{c}\right) \in(0, r) \times U$ such that $\left\|d_{y} \theta\left(t_{c}, y_{c}\right)\right\|<c$. But $T_{X, \bar{\theta}\left(t_{c}, y_{c}\right)} \supset \operatorname{Im} d_{y} \bar{\theta}\left(t_{c}, y_{c}\right)=\operatorname{graph} d_{y} \theta\left(t_{c}, y_{c}\right)$, and from Remark 2.3 we have

$$
\delta\left(\mathbb{R}^{p}, T_{X, \bar{\theta}\left(t_{c}, y_{c}\right)}\right) \leq\left\|d_{y} \theta\left(t_{c}, y_{c}\right)\right\|<c .
$$

This is a contradiction.

Proof of $\operatorname{dim}\left(Y \backslash W_{\mathrm{b}}(X, Y)\right)<p$. By Lemma 2.9 it suffices to prove that $\operatorname{dim}\left(Y \backslash W_{\mathrm{b}^{\prime}}(X, Y)\right)<p$. Define

$$
\psi(y, t)=\sup \left\{\delta\left(\mathbb{R}(x-\pi(x)), T_{X, x}\right): x \in X,\|x-y\| \leq t\right\}, \quad y \in Y, t>0 .
$$

Then $\psi$ is a $\mathcal{D}$-function and there exists $\lim _{t \rightarrow 0^{+}} \psi(y, t)=g(y) \in \mathbb{R}$ for each $y \in Y$. Note that $g: Y \rightarrow \mathbb{R}$ is a $\mathcal{D}$-function and $g(y) \neq 0 \Leftrightarrow y \in W_{\mathrm{b}^{\prime}}(X, Y)$.

If $\operatorname{dim}\left(Y \backslash W_{\mathrm{b}^{\prime}}(X, Y)\right)=p$, then, by Theorem 1.4, there is an open $\mathcal{D}$-set $V^{\prime}$ in $Y$ such that $g>c^{\prime}$ on $V^{\prime}$ for some $c^{\prime}>0$.

Let $S^{\prime}=\left\{x \in X: \delta\left(\mathbb{R}(x-\pi(x)), T_{X, x}\right) \geq c^{\prime}\right\}$. Then $V^{\prime} \subset \bar{S}^{\prime} \backslash S^{\prime}$. So, by Lemma 2.7, there are an open set $U^{\prime} \subset V^{\prime}, \varepsilon>0$ and an analytic $\mathcal{D}$-map $\widetilde{\theta}:(0, \varepsilon) \times U^{\prime} \rightarrow S^{\prime}$ such that $\pi \circ \widetilde{\theta}(t, y)=y$. Fix $y \in U^{\prime}$, define $\gamma(t)=\widetilde{\theta}(t, y)$. Then $\gamma(t) \in X$ and $\pi(\gamma(t))=y$ for all $t \in(0, \varepsilon)$. Applying Lemma 2.10 we have 


$$
\lim _{0^{+}} \delta\left(\mathbb{R}(\gamma(t)-\pi(\gamma(t))), T_{X, \gamma(t)}\right)=0
$$

a contradiction.

2.11. Theorem (Whitney stratification). Let $S_{1}, \ldots, S_{k}$ be $\mathcal{D}$-sets in $\mathbb{R}^{n}$. Then there exists a finite family $\mathcal{W}=\left\{\Gamma_{\alpha}\right\}$ of subsets of $\mathbb{R}^{n}$ satisfying (S1)-(S3) of Corollary 1.5 which has the following property:

(W) If $\Gamma_{\alpha}, \Gamma_{\beta} \in \mathcal{W}, \Gamma_{\beta} \subset \bar{\Gamma}_{\alpha} \backslash \Gamma_{\alpha}$, then $\left(\Gamma_{\alpha}, \Gamma_{\beta}\right)$ satisfies Whitney's conditions a) and b) at all points of $\Gamma_{\beta}$.

Proof. We construct the families $\mathcal{W}^{d}, d=0, \ldots, n$, by decreasing induction on $d$ such that $\mathcal{W}^{d}$ has the following property:

$\left(*_{d}\right) \quad \forall \Gamma_{i}, \Gamma_{j} \in \mathcal{W}^{d}, \Gamma_{j} \subset \bar{\Gamma}_{i} \backslash \Gamma_{i}, \operatorname{dim} \Gamma_{j} \geq d \Rightarrow W_{\mathrm{a}}\left(\Gamma_{i}, \Gamma_{j}\right)=W_{\mathrm{b}}\left(\Gamma_{i}, \Gamma_{j}\right)=\Gamma_{j}$.

Let $\mathcal{W}^{n}$ be a stratification of $\mathbb{R}^{n}$ compatible with $S_{1}, \ldots, S_{k}$ as in Corollary 1.5. Suppose that $\mathcal{W}^{d}$ is constructed $(d \geq 1)$. For every $\Gamma_{j} \in \mathcal{W}^{d}$ with $\operatorname{dim} \Gamma_{j}=d-1$ define

$$
T_{j}=\left(\bigcup\left\{\Gamma_{j} \backslash\left(W_{\mathrm{a}}\left(\Gamma_{i}, \Gamma_{j}\right) \cap W_{\mathrm{b}}\left(\Gamma_{i}, \Gamma_{j}\right)\right): \Gamma_{i} \in \mathcal{W}^{d}, \Gamma_{j} \subset \bar{\Gamma}_{i} \backslash \Gamma_{i}\right\}\right) \cap \Gamma_{j} .
$$

Note that $\operatorname{dim} T_{j}<\operatorname{dim} \Gamma_{j}$ by Theorem 2.5 .

Let $\mathcal{V}^{d}$ be a stratification of $\mathbb{R}^{n}$ into cells which is compatible with $\Gamma_{j} \backslash T_{j}, T_{j}\left(\Gamma_{j} \in \mathcal{W}^{d}\right.$, $\left.\operatorname{dim} \Gamma_{j}=d-1\right)$ and $\Gamma_{l}\left(\Gamma_{l} \in \mathcal{W}^{d}, \operatorname{dim} \Gamma_{l}<d-1\right)$ (such a stratification exists by Corollary 1.5). Define $\mathcal{W}^{d-1}=\left\{\Gamma \in \mathcal{W}^{d}: \operatorname{dim} \Gamma \geq d\right\} \cup\left\{\Gamma \in \mathcal{V}^{d}: \operatorname{dim} \Gamma \leq d-1\right\}$. Then $\mathcal{W}^{d-1}$ satisfies $\left(*_{d-1}\right)$. The family of cells $\mathcal{W}=\mathcal{W}^{0}$ is the desired stratification

Since Whitney stratified spaces can be triangulated (see, for example, [3]), Theorem 2.11 implies

2.12. Corollary (triangulation). Let $S \subset \mathbb{R}^{n}$ be a $\mathcal{D}$-set and $S_{1}, \ldots, S_{k}$ be $\mathcal{D}$-sets contained in $S$. Then $S$ admits a triangulation compatible with $S_{1}, \ldots, S_{k}$, i.e. there exist a simplicial complex $K$ and a homeomorphism $h:|K| \rightarrow S$ such that each $S_{i}, i=1, \ldots, k$, is a union of some elements of $\{h(\sigma): \sigma \in K\}$.

Note that in [1] van den Dries proved that $h$ can be taken to be a $\mathcal{D}$-function.

Acknowledgements. The author would like to thank Prof. Wiesław Pawłucki and the Institute of Mathematics of the Jagiellonian University, Kraków.

\section{References}

[1] L. van den Dries, Tame topology and O-minimal structures, mimeographed notes (1991).

[2] L. van den Dries and C. Miller, The field of reals with restricted analytic functions and unrestricted exponentiation, Israel J. Math. (1991).

[3] R. M. Goresky, Triangulation of stratified objects, Proc. Amer. Math. Soc. 72 (1978), 193-200.

[4] A. G. Khovanskiŭ, Fewnomials, Transl. Math. Monographs 88, Amer. Math. Soc., 1991.

[5] T. L. Loi, thesis, Jagiellonian University, Kraków 1993. 
[6] T. L. Loi, Analytic cell decomposition of sets definable in the structure $\mathbb{R}_{\mathrm{exp}}$, Ann. Polon. Math. 59 (1994), 255-266.

[7] - - On the global Eojasiewicz inequalities for the class of analytic logarithmico-exponential functions, C. R. Acad. Sci. Paris Sér. I 318 (1994), 543-548.

[8] S. Łojasiewicz, Ensembles Semi-Analytiques, I.H.E.S., Bures-sur-Yvette, 1965.

[9] A. J. Wilkie, Some model completeness results for expansions of the ordered field of real numbers by Pfaffian functions, preprint, 1991.

[10] - Model completeness results for expansions of the real field II: The exponential function, manuscript, 1991. 